\title{
Ultrasound-guided transversalis fascia plane block versus transmuscular quadratus lumborum block for post-operative analgesia in inguinal hernia repair
}

\author{
Ahmed Zaghloul Fouad ${ }^{1}$, Iman Riad M. Abdel-Aal ${ }^{1}$, Mohamed Rabie Mohamed Ali Gadelrab ${ }^{2}$, and \\ Hany Mohammed El-Hadi Shoukat Mohammed ${ }^{1}$
}

'Department of Anesthesia, Surgical Intensive Care and Pain Management, Cairo University, Cairo, Egypt

${ }^{2}$ Department of Anesthesia, Agouza Police Hospital, Giza, Egypt

Received October 26, 2020

Revised December 16, 2020

Accepted December 22, 2020

Handling Editor: Young Hoon Kim

\section{Correspondence}

Hany Mohammed El-Hadi Shoukat Mohammed

Surgical Intensive Care and Pain Management, Faculty of Medicine, Cairo University, El Saray street, El Manial, Cairo 11956, Egypt

Tel: +201003066806

Fax: +20223644383

E-mail: oblfollower_2001@yahoo.com
Background: Inguinal hernia repair is one of the most commonly performed surgical procedures. Regional blocks might provide excellent analgesia and reduce complications in the postoperative period. We aimed to compare the postoperative analgesic effect of the ultrasound-guided transversalis fascia (TF) plane block versus the transmuscular quadratus lumborum $(\mathrm{QL})$ block in patients undergoing unilateral inguinal hernia repair.

Methods: Fifty patients enrolled in this comparative study and were randomly assigned into two equal groups. One group received an ultrasound-guided QL block. In comparison, the other group received an ultrasound-guided TF plane block. The primary outcome was the patient-assessed resting, and movement-induced pain on the numeric pain rating scale (NRS) measured at 30 minutes postoperatively. Secondary outcomes included the percentage of patients receiving rescue analgesia in the first postoperative day, ease of performance of the technique, and incidence of adverse effects.

Results: There were no statistically significant differences in NRS at rest and with movement between the groups over the first 24 hours postoperatively. The proportion of patients that received postoperative rescue analgesics during the first 30 minutes postoperatively was $4 \%(n=1)$ in the QL group compared to $12 \%(n=3)$ in the TF group. However, the mean performance time of the TF block was shorter than that of the QL block, and the performance of the TF block appeared easier technically.

Conclusions: The ultrasound-guided TF plane block could be as effective as the QL block in lowering pain scores and decreasing opioid consumption following nonrecurrent inguinal herniorrhaphy.

Key Words: Analgesia; Analgesics, Opioid; Fascia; Hernia, Inguinal; Herniorrhaphy; Nerve Block; Pain Management; Pain, Postoperative; Ultrasonography, Interventional.

\section{INTRODUCTION}

Inguinal hernia repair is one of the most common surgical procedures in the world. Annually, more than 20 million inguinal hernia repairs are conducted worldwide [1].

Uncontrolled postoperative pain increases the incidence (c) This is an open-access article distributed under the terms of the Creative Commons Attribution Non-Commercial License (http://creativecommons.org/licenses/by-nc/4.0/), which permits unrestricted non-commercial use, distribution, and reproduction in any medium, provided the original work is properly cited.

(c) The Korean Pain Society, 2021
Author contributions: Ahmed Zaghloul Fouad: Methodology; Iman Riad M. Abdel-Aal: Methodology; Mohammed Rabie Mohamed Ali Gadelrab: Investigation; Hany Mohammed El-Hadi Shoukat Mohammed: Writing/ manuscript preparation. 


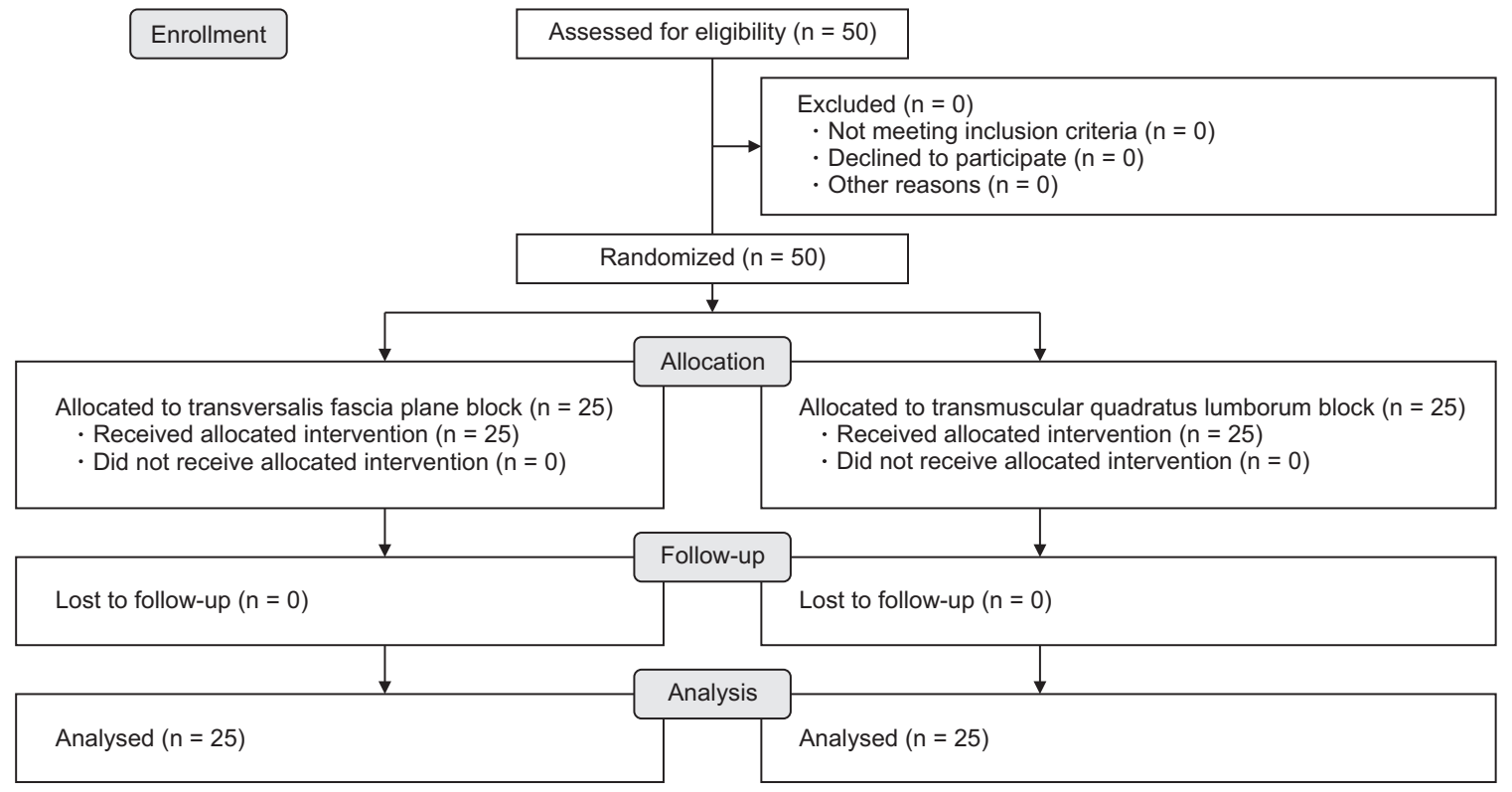

Fig. 1. Flowchart of the study.

of postoperative complications. Regional blocks, as a part of multimodal analgesia, can improve pain control in the postoperative period and reduce complications that may arise from using a single mode of analgesia [2]. For example, reliance on opioid analgesia increases the incidence of pruritus, nausea, and vomiting, as well as respiratory depression [3].

Hebbard [4] first described the ultrasound (US)-guided transversalis fascia plane (TFP) block in 2009. A local anesthetic (LA) injected between the transversus abdominis muscle and its deep investing fascia will block the anterior and the lateral branches of the T12 and L1 nerves [4].

Blanco in 2007 described the US-guided quadratus lumborum (QL) block as a modification of the transversus abdominis plane (TAP) block. LA in the QL block will spread into the thoracic paravertebral space to produce blockade of the abdominal wall. This spread will anesthetize the lateral and anterior cutaneous branches from T7 to L1 [5].

The transmuscular approach of the QL block (QLB-3) is regarded as a simple and safer alternative to the anterior approach of the original QL block [6].

In this study, we tried to compare the effectiveness of the US-guided TFP block versus the transmuscular QL block in providing postoperative analgesia in patients undergoing unilateral inguinal hernia repair.

\section{MATERIALS AND METHODS}

\section{Study design}

This prospective comparative study was conducted after receiving Departmental and Faculty Ethics Committee approval (N-48-2018). The study was registered at ClinicalTrials.gov (with a Trial Identifier: NCT04026243). A CONSORT checklist for enrollment and allocation of patients is illustrated in (Fig. 1). Before the commencement of the study, informed consent was obtained from each patient for participation in the study. Fifty patients with American Society of Anesthesiologists physical status I and II, aged above 18 years old, who had undergone nonrecurrent unilateral inguinal hernia repair at Kasr Alaini Hospital, were enrolled in the study. The exclusion criteria were body mass index $>35 \mathrm{~kg} / \mathrm{m}^{2}$, patients with difficulty in evaluating their level of pain, coagulopathy, presence of skin infection or hematoma in the vicinity of the puncture site, and known allergy to any of the study drugs.

\section{Anesthesia application}

In the operation theatre, an 18-gauge intravenous (IV) catheter was placed, and monitoring devices were attached that included electrocardiograph, pulse oximetry (arterial oxygen saturation), and non-invasive blood pressure using GE Datex Ohmeda Monitor (GE-M0031; GE Healthcare, Madison, WI). Emergency drugs and equipment were ready and prepared. The numeric pain rating scale (NRS) was explained clearly, to all patients before induction of anesthesia. General anesthesia (GA) was induced with fentanyl (2 mcg/ $\mathrm{kg})$, propofol $(1.5-2.5 \mathrm{mg} / \mathrm{kg})$, and atracurium besylate $(0.5 \mathrm{mg} / \mathrm{kg})$. An endotracheal tube (ETT) was inserted, and controlled ventilation was adjusted to maintain normocapnia. Anesthesia was maintained with sevoflurane at $2 \%-3 \%$ and boluses of atracuri- 


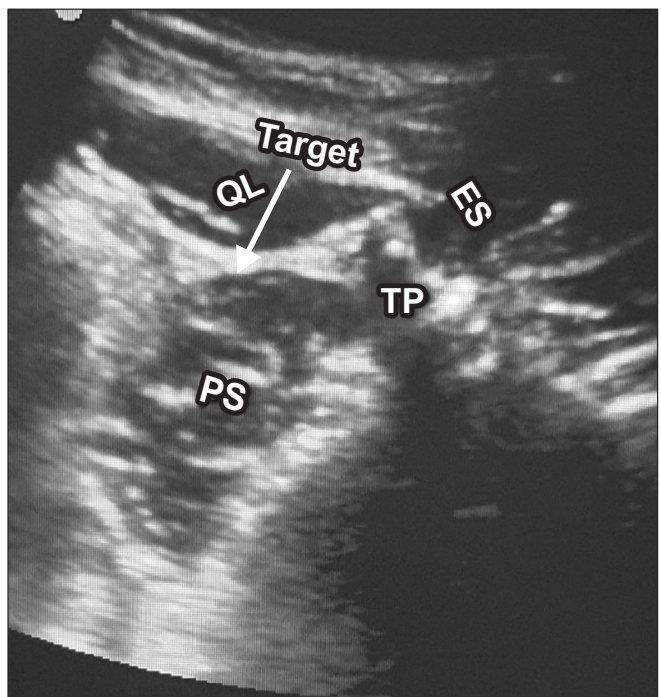

Fig. 2. Sonoanatomy for QL3 block. QL: quadratus lumborum muscle, PS: psoas major muscle, ES: erector spinae muscle, TP: transverse process.

um $(0.1 \mathrm{mg} / \mathrm{kg})$ every 30 minutes. A prophylactic antibiotic and antiemetic were given after induction of GA.

\section{Patient randomization}

Patients were randomly assigned using computer-generated numbers and concealed sequentially numbered, sealed opaque envelopes, divided into two equal groups of twenty-five patients each. US-guided transmuscular QL blocks (QLB-3) were performed in Group (QL) while US-guided TFP blocks were performed in Group (TF).

\section{Block interventions}

All blocks were performed on patients, following GA induction and ETT insertion, under the guidance of a digital ultrasonic diagnostic imaging system (Mindray DP30 Ultrasound Machine; Mindray Medical International Ltd., Shenzhen, China), using a low frequency (2-6 MHz) curvilinear probe and a 100-150 mm short-bevel $22 \mathrm{G}$ echogenic needle. Before US scanning, the operator wore a sterile gown and gloves following the routine scrubbing. The flank skin was prepared with antiseptic solution, and fenestrated draping and dressings were used for all procedures. A sterile gel and US probe cover were applied to the US probe before scanning.

The QL group ( $\mathrm{n}=25)$ : Patients were placed in the lateral position with the side to be anesthetized turned upwards. The US probe was placed in the transverse plane at the abdominal flank immediately cranial to the iliac crest. The transducer was then moved dorsally, keeping the transverse orientation until the QL muscle was identified with

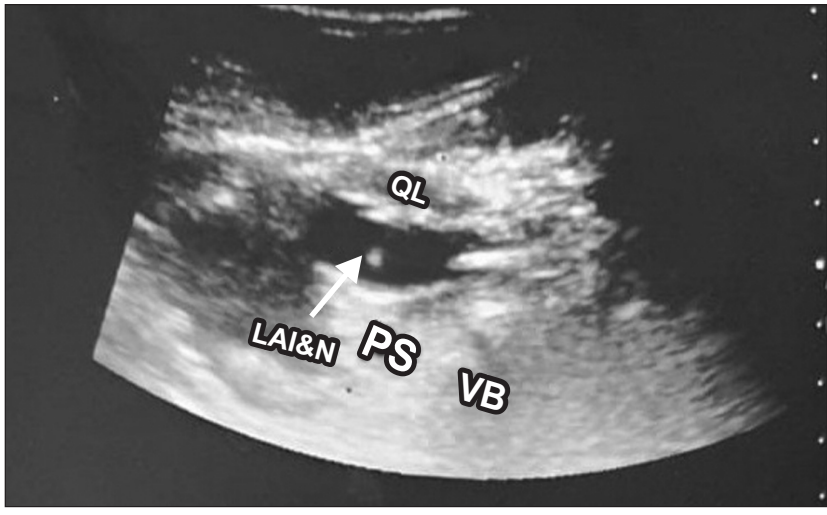

Fig. 3. Picture showing spread of local anesthetic injectate in quadratus lumborum muscle (QL) block. Arrow pointing to target for local anesthetic solution deposition. LAI\&N: local anesthetic injectate and needle tip, PS: psoas major muscle, VB: vertebral body.

its attachment to the lateral edge of the transverse process of the L4 vertebral body. A clearly recognizable pattern of a shamrock with the three leaves made by the psoas major muscle (PS) anteriorly, the erector spinae muscle posteriorly, and the QL muscle adherent to the apex of the transverse process, were seen (Fig. 2). The needle was inserted in-plane to the transducer, and the tip of the needle advanced through the QL muscle, penetrating its fascia. Once the tip of the needle was correctly placed between the QL and PS, a negative aspiration was confirmed, and then $2 \mathrm{~mL}$ of normal saline was instilled to establish the correct separation of the plane. Then, $30 \mathrm{~mL}$ of $0.25 \% \mathrm{bu}-$ pivacaine (Sunnypivacaine ${ }^{\circledR} 0.5 \%, 20 \mathrm{~mL}$; Sunny Pharmaceutical, Badr, Egypt) was injected (Fig. 3).

The TF group ( $\mathrm{n}=25)$ : The patient was placed in the lateral position with the side to be anesthetized upwards, with the investigator facing the patient. The probe was placed on the midaxillary line, just cephalad to the iliac crest. Scanning started anteriorly, identifying the three muscles of the anterior abdominal wall. The transversus abdominis was identified as a hyper-echoic aponeurosis passing anterolateral to the QL (Fig. 4). The scan continued posteriorly to visualize the kidney, liver, or viscera deep to the transversus abdominis to avoid their injury. The needle was inserted anterior to the US probe using an inplane approach posterolaterally through the three lateral abdominal muscles. After correct placement of the needle between the transversus abdominis muscle and the transversalis fascia, and negative aspiration, $2 \mathrm{~mL}$ of normal saline was instilled for hydro-dissection of the plane. Then, $30 \mathrm{~mL}$ of $0.25 \%$ bupivacaine (Sunnypivacaine ${ }^{\circledR} 0.5 \%, 20$ mL; Sunny Pharmaceutical) was injected (Fig. 5).

Patients then returned to the supine position, and surgery commenced. After skin closure, inhaled anesthesia was discontinued and reversal of muscle relaxation with 


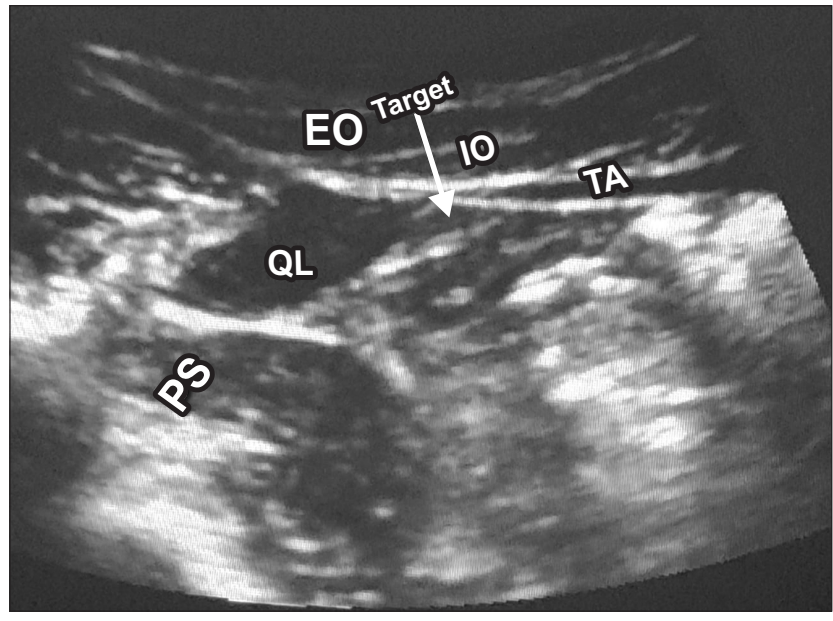

Fig. 4. Sonoanatomy for transversalis fascia plane block. EO: external oblique muscle, IO: internal oblique muscle, TA: transversus abdominis muscle, QL: quadratus lumborum muscle, PS: psoas major muscle.

atropine $(0.02 \mathrm{mg} / \mathrm{kg})$ and neostigmine $(0.05 \mathrm{mg} / \mathrm{kg})$ were administered intravenously after the return of the patient's spontaneous breathing. The patient was then transferred to the post-anesthesia care unit (PACU) for complete recovery and monitoring.

A block was considered a failed block when the patient required more than one dose of rescue analgesia $(5 \mathrm{mg}$ of nalbuphine) in the first hour postoperatively.

\section{Evaluation of pain}

We used the NRS for the evaluation of both resting and movement-induced postoperative pain. This NRS is an 11-point scale for pain assessment started from 0 to 10 , where 0 means 'no pain' and 10 indicates the 'worst pain imaginable'. NRS scores were recorded at 10, 30, 60, and 90 minutes after surgery and at 24 hours postoperatively.

\section{Postoperative analgesia}

All patients were given $1 \mathrm{~g}$ of IV paracetamol before the end of surgery. In the PACU, rescue analgesia in the form of IV nalbuphine (in $5 \mathrm{mg}$ increments) was given when the NRS score was more than 4 in the immediate postoperative period. In the ward, rescue analgesia in the form of IV nalbuphine (in $5 \mathrm{mg}$ increments) was administered according to the NRS assessment with an emphasis on not exceeding the maximal dose of $60 \mathrm{mg}$ per 24 hours.

\section{Outcome measure}

The primary outcome was the postoperative patientassessed resting and movement-induced pain on the NRS, measured at 30 minutes postoperatively (the higher score

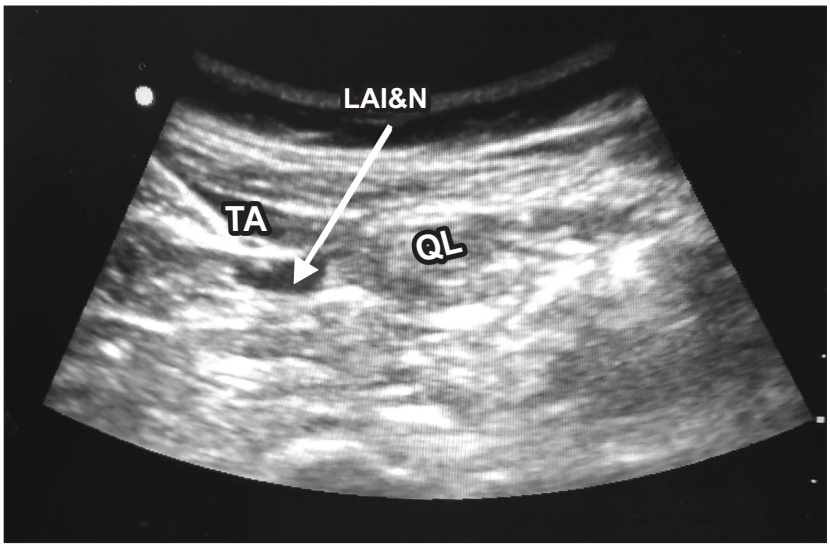

Fig. 5. Picture showing spread of local anesthetic solution and needle tip for transversalis fascia plane block. Arrow pointing to target for local anesthetic solution deposition. TA: transversus abdominis muscle, QL: quadratus lumborum muscle, LAI\&N: local anesthetic injectate and needle tip.

was taken). We also recorded the number of increments of nalbuphine rescue analgesia needed in the immediate postoperative period from 30 minutes up to 24 hours postoperatively. The level of sensory block was assessed at 10 minutes after PACU admission (comparing the nerve territories on the surgery side to the contralateral territory by application of a cold, wet cotton swab). Sensitivity to cold was graded on a scale of 0 to 2 (2: normal sensitivity to cold; 1: hypoesthesia; and 0: anesthesia).

Block performance time in minutes (from probe contact with the skin until the needle was withdrawal), and ease of the performance of the block were recorded. The ease of the performance of the block was assessed by asking the operator to rate the procedure on a simple verbal scale (easy/moderately difficult/difficult). The block was rated as an "easy block" when the successful block (adequate hydrodissection under US) was achievied after the first skin puncture with no need for needle image optimization (no adjustment of depth, gain, or focus to visualize the needle path). A "moderately difficult block" was recorded when a successful block was achieved after more than one skin puncture attempt or when needle image optimization was needed. We recorded the procedure as a "difficult block" when a successful block was completed after more than one skin puncture attempt and with the need for needle image optimization. Patient satisfaction regarding pain management was recorded 6 hours after surgery by a verbal questionnaire ("How did you find your pain sensation in the past 6 hours?"). The patient responded to the questionnaire by selecting the answer from being very satisfied, satisfied, not very satisfied, or dissatisfied. Incidence of adverse effects, such as postoperative nausea and vomiting, urinary retention, and LA toxicity was reported postoperatively for up to 24 hours. We measured duration of 
surgery (time in minutes from skin incision to skin closure for up to 180 minutes after GA induction) and duration of GA (time in minutes from GA induction to the removal of the ETT). Heart rate (HR), systolic blood pressure (SBP), and mean arterial pressure (MAP) were also recorded. Measurements were taken at the following intervals: 1 minute before induction of anesthesia $\left(\mathrm{T}_{0}\right), 5$ minutes after induction of anesthesia $\left(\mathrm{T}_{1}\right), 5$ minutes after injection of LA $\left(\mathrm{T}_{2}\right), 15$ minutes after injection of LA $\left(\mathrm{T}_{3}\right), 30$ minutes after injection of LA $\left(\mathrm{T}_{4}\right), 45$ minutes after injection of LA $\left(\mathrm{T}_{5}\right), 5$ minutes after removal of the ETT $\left(\mathrm{T}_{6}\right), 15$ minutes after removal of the ETT $\left(\mathrm{T}_{7}\right), 30$ minutes after removal of the ETT $\left(\mathrm{T}_{8}\right)$, and 60 minutes after removal of the ETT $\left(\mathrm{T}_{9}\right)$.

\section{Sample size calculation}

A power calculation estimated that to detect an effect size of 1.15 for the pain score between the two studied groups (QL block versus TAP block) [7], with a $P$ value $<0.05$ and $80 \%$ power, confidence level 0.95 , a sample size of 22 patients for each group was needed. However, 50 patients were included in this research to deal with the non-response rate ( 25 for each group). This calculation was made by using the G Power 3.1.

\section{Statistical analysis}

Study variables were first analyzed using descriptive techniques. Continuous variables were defined using the mean as the measure of central tendency, and the standard deviation as the measure of dispersion. Qualitative variables were expressed as absolute numbers and percentages. Following this, study groups (TF and QL) were compared by means of bivariate analysis. For the continuous dependent

Table 1. Patient data and clinical characteristics of the studied groups

\begin{tabular}{lccc}
\hline Characteristic & QL group $(\mathrm{n}=25)$ & TF group $(\mathrm{n}=25)$ & $P$ value \\
\hline Age $(\mathrm{yr})$ & $29.9 \pm 6.4$ & $31.6 \pm 7.2$ & 0.281 \\
Sex & & & 0.155 \\
$\quad$ Male & $23(92.0)$ & $25(100.0)$ & \\
$\quad$ Female & $2(8.0)$ & $0(0.0)$ & \\
BMI $\left(\mathrm{kg} / \mathrm{m}^{2}\right)$ & $30.1 \pm 2.2$ & $29.9 \pm 1.3$ & 0.611 \\
Side of surgery & & & 0.779 \\
$\quad$ Left & $14(56.0)$ & $15(60.0)$ & \\
$\quad$ Right & $11(44.0)$ & $10(40.0)$ & \\
ASA score & & & 0.739 \\
ASA I & $20(80.0)$ & $21(84.0)$ & \\
ASA II & $5(20.0)$ & $4(16.0)$ & \\
\hline
\end{tabular}

Values are presented as mean \pm standard deviation or number (\%). $P$ value $<0.05$ is considered statistically significant.

QL: quadratus lumborum block, TF: transversalis fascia plane block, BMI: body mass index, ASA: American Society of Anesthesiologists. variable, normality was evaluated using the Shapiro-Wilk test. Normally distributed variables were evaluated using the Student's $t$-test or analysis of variance. The MannWhitney test or Kruskal-Wallis test were used to analyze non-parametric data. Categorical variables were analyzed using the chi-square test. A $P$ value of $<0.05$ was considered statistically significant. All statistical calculations were performed using SPSS ver. 16.0 (SPSS Inc., Chicago, IL).

\section{RESULTS}

A total of fifty patients were screened for eligibility and enrolled in the study after randomization (Fig. 1). Patient data and clinical characteristics of the studied groups are shown in Table 1. These results were comparable between both groups, with no statistically significant difference.

The mean postoperative NRS score at rest at 30 minutes was $3.64 \pm 0.63$ in the QL group compared to $3.80 \pm 0.95$

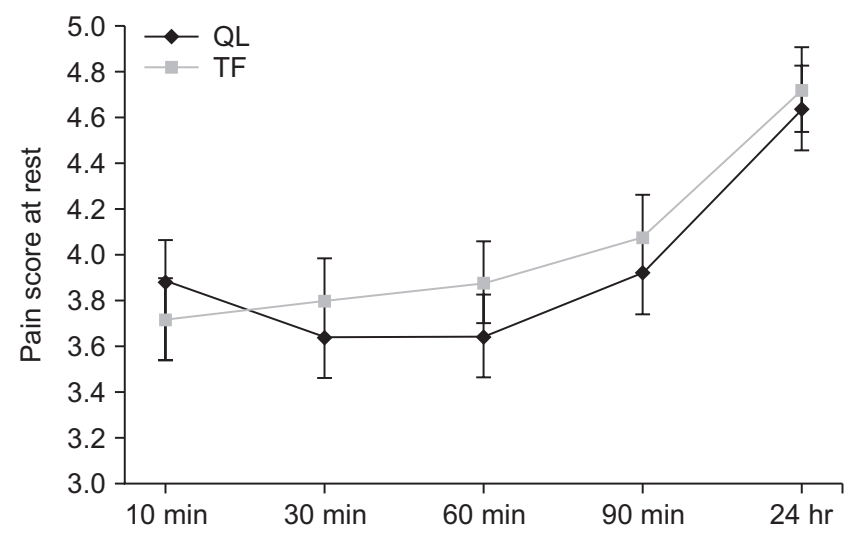

Fig. 6. Postoperative numerical pain rating score at rest. Data presented as mean \pm standard deviation. QL: quadratus lumborum block, TF: transversalis fascia plane block.

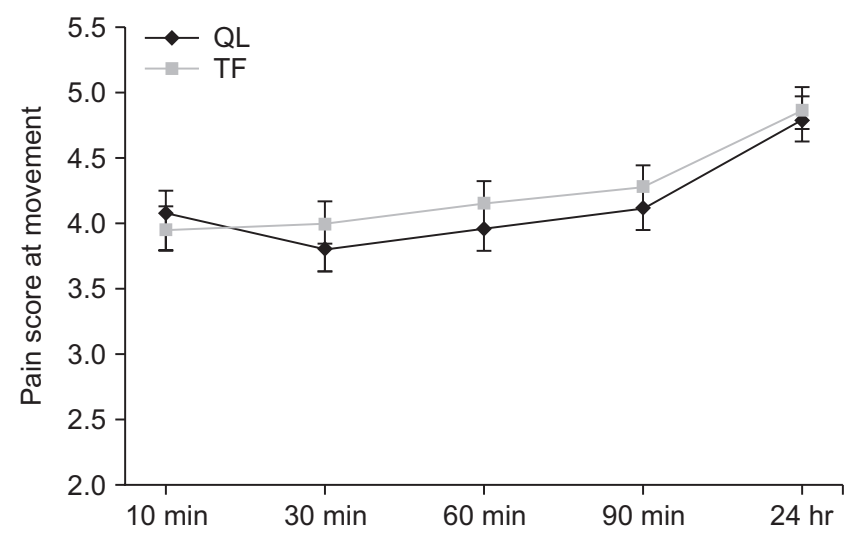

Fig. 7. Postoperative numerical pain rating score with leg movement. Data presented as mean \pm standard deviation. QL: quadratus lumborum block, TF: transversalis fascia plane block. 


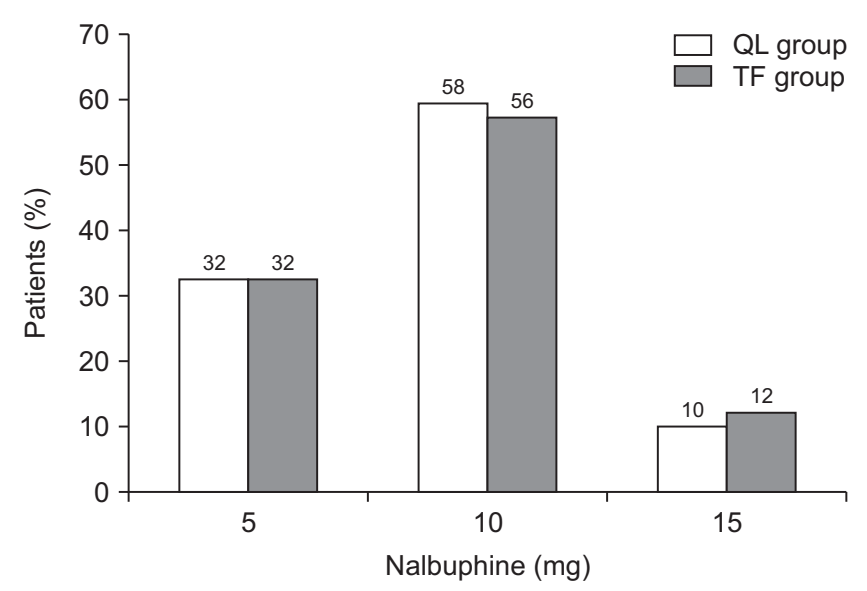

Fig. 8. Percentage of patients receiving postoperative rescue analgesia. QL: quadratus lumborum block, TF: transversalis fascia plane block.

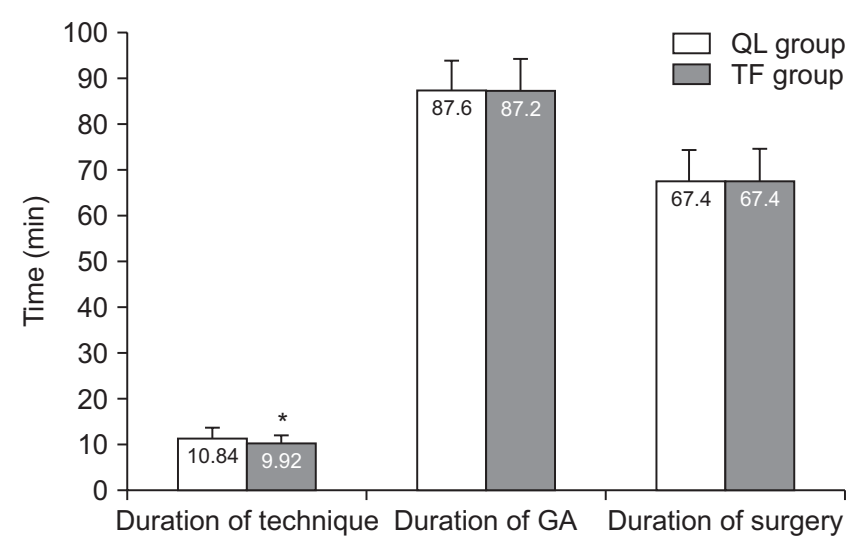

Fig. 9. Duration of general anesthesia (GA), surgery, and block performance time. The error bars indicate the standard deviation. QL: quadratus lumborum block, TF: transversalis fascia plane block. *Means statistically significant difference.

in the TF group. Both groups showed no statistically significant differences in NRS scores either at rest or with leg movement over the first 24 hours postoperatively (Figs. 6, 7).

Although $12 \%$ of patients (3/25) in the TF group received rescue analgesia at 30 minutes postoperatively compared to $4 \%(1 / 25)$ in the QL group, this difference was not statistically significant. Most patients in both groups received two doses of rescue analgesia (i.e., $10 \mathrm{mg}$ of IV nalbuphine) during the first 24 hours, postoperatively. However, these results were comparable between both groups, with no statistically significant difference (Fig. 8).

The mean performance time of the block was $9.92 \pm 1.76$ minutes in the TF group, which was statistically significantly shorter $(P=0.028)$ when compared to that in the QL group, which was $10.84 \pm 1.97$. Block performance time, duration of surgery, and duration of GA are illustrated in Fig. 9.
Table 2. Easiness of performance of the technique and patient satisfaction

\begin{tabular}{lrrr}
\hline \multicolumn{1}{c}{ Characteristic } & $\begin{array}{r}\text { QL group } \\
(\mathrm{n}=25)\end{array}$ & $\begin{array}{r}\text { TF group } \\
(\mathrm{n}=25)\end{array}$ & P value \\
\hline $\begin{array}{l}\text { Ease of performance of the technique } \\
\text { Easy }\end{array}$ & \multicolumn{3}{c}{$0.031^{*}$} \\
$\quad 14(56.0)$ & $21(84.0)$ & \\
$\quad$ Moderately difficult & $11(44.0)$ & $4(16.0)$ & \\
$\begin{array}{l}\text { Patient satisfaction } \\
\text { Very satisfied }\end{array}$ & $19(76.0)$ & $21(84.0)$ & 0.489 \\
$\quad$ Satisfied & $6(24.0)$ & $4(16.0)$ & \\
\hline
\end{tabular}

Values are presented as number (\%). $P$ value $<0.05$ is considered statistically significant.

QL: quadratus lumborum block, TF: transversalis fascia plane block. $* P<0.05$ statistically significant difference.

The incidence of a successful block from the first trial after skin puncture without needle or image optimization was achieved more easily in the TF group with a statistically significant difference $(P=0.031)$. Ease of performance of the technique and patient satisfaction are shown in Table 2.

All patients (100\%) in both groups had a sensory level at the T12-L1 dermatomal level as assessed in the immediate postoperative period. Unfortunately, grading by cold sensitivity at the neural level was difficult to achieve, as all patients were unable to discriminate between anesthesia and hypoesthesia.

Both the QL block and TF block were considered to have failed when the patient required more than two doses of rescue analgesia in the first hour, postoperatively. Fortunately, according to this definition, the incidence of "failed block" was zero percent in both groups, and there was no statistically significant difference. Patient satisfaction with postoperative pain perception was demonstrated in Table 2.

Changes in HR, SBP, and MAP were comparable between the groups, with no statistically significant difference. There were no reported perioperative complications in either group.

\section{DISCUSSION}

The present study showed that the TFP block is equally effective as the QL block in controlling postoperative pain in inguinal hernia repair. Block performance time in the TF group was shorter than in the QL group, and the TFP block was an easier block to perform. To the best of our knowledge, this is the first study conducted to compare the analgesic efficacy of TFP block to the transmuscular QL block in inguinal hernia repair surgery.

Although LA deposited at different fascial planes in both 
types of blocks, the comparable analgesic efficacy in this study between both groups could be attributed to efficient LA spread to the iliohypogastric and ilioinguinal nerves (T12, L1) but by different mechanisms. Several studies [812] have shown that abdominal wall blocks significantly reduced postoperative pain and consumption of opioids in the first 24 postoperative hours, and they were associated with fewer adverse events. These results are consistent with our findings that revealed that postoperative pain management was excellent in both groups, with no reported adverse events, and patients being very satisfied with pain management.

López-González et al. [13] conducted a similar study that compared the US-guided TFP block to the anterior TAP block in outpatient inguinal hernia repair. They concluded that the resting and movement-induced pain scores were similar in both groups. Although they studied the TAP block rather than the QL block, they yielded parallel results to the current study. This similarity could be attributed to the fact that the QL block is considered to be a posterior approach or extension to the TAP block.

Scimia et al. [14] published a case report in which they used the US-guided TFP block as an approach for anesthesia for a herniorrhaphy case. The authors declared that the US-guided TFP block could provide a reliable block of the nerves in T12-L1. Moreover, they suggested that this block presented a viable alternative to US-guided anterior TAP and QL blocks and general and neuraxial anesthesia. And if given as a part of a multimodal analgesia regimen, it can reduce opioid requirements with excellent patient satisfaction.

Rahimzadeh et al. [15], in a randomized study, compared the US-guided TFP and TAP block on postoperative pain in patients undergoing elective cesarean section. Although their study population was different and they compared the TAP block instead of the QL block to the TFP block, yet they reported similar results in some ways to the present study. For instance, they found that there was no difference between the TAP and TFP blocks in pain severity at rest and while coughing, or in nausea, and vomiting, and satisfaction rate; both methods had the proper pain control effect with neither method being superior.

Ahmed et al. [16] conducted a comparative study between the posterior QL block (QLB-2) and transmuscular QL block (QLB-3) in patients who underwent surgical repair of unilateral inguinal hernia. They found that the transmuscular approach (QLB-3) produced a greater postoperative analgesic effect with less postoperative opioid consumption compared to the posterior approach (QLB-2). However, they used only $20 \mathrm{~mL} 0.25 \%$ bupivacaine compared to the $30 \mathrm{~mL}$ used in our study.

Öksüz et al. [17] compared the QL block to the TAP block in controlling postoperative pain following lower abdominal surgery. They found that the number of patients who required analgesia in the first 24 hours postoperatively was significantly lower in the QL block group than in the TAP block group. Also, satisfaction scores were higher in the QL block group compared with the TAP block group, with no reported postoperative complications in either group.

In our study, we used $30 \mathrm{~mL}$ of bupivacaine as the volume for LA injectate in both regional blocks. In the literature, there is no consensus on the type, concentration, and amount of LA used to perform the QL block. The QL can be blocked by the injection of $15-30 \mathrm{~mL}(0.2-0.4 \mathrm{~mL} / \mathrm{kg})$ of a LA on the left and right side of the abdominal wall with $0.125 \%-0.375 \%$ bupivacaine, levobupivacaine, or ropivacaine [18].

As we used the same LA volume for both blocks and the injection for both the QL block and TFP block are quite near each other, we expected these similar results. Carline et al. [19] compared the spread of dye and nerve involvement after three different QL blocks and found that all the transmuscular QL blocks (QLB-3) spread consistently to the L1 and L3 nerve roots. Also, Yang et al. [20] compared the spread of dye between different needle approaches for US-guided QL blocks in cadavers. They found that the thoracolumbar fascia was deeply stained in all blocks, however the dye stained the transversus abdominis and TFP in the alternative QL approach [20].

Not calculating of the total duration of the block and the absence of a control group receiving a placebo injection could be considered limitations to this study. Further studies should be conducted on a larger number of patients using different drugs and concentrations, to study the efficacy of catheter infusion on postoperative pain management in these types of patients and to follow the effect of these regional blocks on the development of chronic post-herniorrhaphy groin pain.

We concluded that the US-guided TFP block could be as influential as the QL block in lowering pain scores and decreasing opioid consumption following non-recurrent inguinal herniorrhaphy. However, the US-guided TFP block could be technically more comfortable and faster. Further studies are needed to support these findings.

\section{ACKNOWLEDGMENTS}

The authors would like to thank Dr. Mohammed Ali, MD, Department of Community and Public Health, Faculty of Medicine, Cairo University, for his significant contribution to statistical analysis for this work. 


\section{CONFLICT OF INTEREST}

No potential conflict of interest relevant to this article was reported.

\section{FUNDING}

No funding to declare.

\section{ORCID}

Ahmed Zaghloul Fouad, https://orcid.org/0000-0003-1012-2549

Iman Riad M. Abdel-Aal, https://orcid.org/0000-0002-1933-0822

Mohamed Rabie Mohamed Ali Gadelrab,

https://orcid.org/0000-0002-1249-0823

Hany Mohammed El-Hadi Shoukat Mohammed, https://orcid.org/0000-0003-0736-2218

\section{REFERENCES}

1. Aasvang E, Kehlet H. Surgical management of chronic pain after inguinal hernia repair. Br J Surg 2005; 92: 795-801.

2. Chou R, Gordon DB, de Leon-Casasola OA, Rosenberg JM, Bickler S, Brennan T, et al. Management of postoperative pain: a clinical practice guideline from the American Pain Society, the American Society of Regional Anesthesia and Pain Medicine, and the American Society of Anesthesiologists' Committee on Regional Anesthesia, Executive Committee, and Administrative Council. J Pain 2016; 17: 131-57.

3. Bulka CM, Shotwell MS, Gupta RK, Sandberg WS, Ehrenfeld JM. Regional anesthesia, time to hospital discharge, and inhospital mortality: a propensity score matched analysis. Reg Anesth Pain Med 2014; 39: 381-6.

4. Hebbard PD. Transversalis fascia plane block, a novel ultrasound-guided abdominal wall nerve block. Can J Anaesth 2009; 56: 618-20.

5. Chakraborty A, Goswami J, Patro V. Ultrasound-guided continuous quadratus lumborum block for postoperative analgesia in a pediatric patient. A A Case Rep 2015; 4: 34-6.

6. Hansen CK, Dam M, Bendtsen TF, Børglum J. Ultrasoundguided quadratus lumborum blocks: definition of the clinical relevant endpoint of injection and the safest approach. A A Case Rep 2016; 6: 39.

7. Kumar GD, Gnanasekar N, Kurhekar P, Prasad TK. A comparative study of transversus abdominis plane block versus quadratus lumborum block for postoperative analgesia following lower abdominal surgeries: a prospective double- blinded study. Anesth Essays Res 2018; 12: 919-23.

8. Webster K. The Transversus Abdominis Plane (TAP) block: abdominal plane regional anaesthesia. Update Anaesth 2008; $24: 24-9$

9. Carney J, McDonnell JG, Ochana A, Bhinder R, Laffey JG. The transversus abdominis plane block provides effective postoperative analgesia in patients undergoing total abdominal hysterectomy. Anesth Analg 2008; 107: 2056-60.

10. El-Dawlatly AA, Turkistani A, Kettner SC, Machata AM, Delvi MB, Thallaj A, et al. Ultrasound-guided transversus abdominis plane block: description of a new technique and comparison with conventional systemic analgesia during laparoscopic cholecystectomy. Br J Anaesth 2009; 102: 763-7.

11. Carney J, Finnerty O, Rauf J, Curley G, McDonnell JG, Laffey JG. Ipsilateral transversus abdominis plane block provides effective analgesia after appendectomy in children: a randomized controlled trial. Anesth Analg 2010; 111: 998-1003.

12. Aveline C, Le Hetet H, Le Roux A, Vautier P, Cognet F, Vinet E, et al. Comparison between ultrasound-guided transversus abdominis plane and conventional ilioinguinal/iliohypogastric nerve blocks for day-case open inguinal hernia repair. $\mathrm{Br}$ J Anaesth 2011; 106: 380-6.

13. López-González JM, López-Álvarez S, Jiménez Gómez BM, Areán González I, Illodo Miramontes G, Padín Barreiro L. Ultrasound-guided transversalis fascia plane block versus anterior transversus abdominis plane block in outpatient inguinal hernia repair. Rev Esp Anestesiol Reanim 2016; 63: 498-504.

14. Scimia P, Basso Ricci E, Petrucci E, Behr AU, Marinangeli F, Fusco P. Ultrasound-guided transversalis fascia plane block: an alternative approach for anesthesia in inguinal herniorrhaphy: a case report. A A Pract 2018; 10: 209-11.

15. Rahimzadeh P, Faiz SHR, Imani F, Rahimian Jahromi M. Comparison between ultrasound guided transversalis fascia plane and transversus abdominis plane block on postoperative pain in patients undergoing elective cesarean section: a randomized clinical trial. Iran Red Crescent Med J 2018; 20: e67844.

16. Ahmed A, Fawzy M, Nasr MAR, Hussam AM, Fouad E, Aboeldahb $\mathrm{H}$, et al. Ultrasound-guided quadratus lumborum block for postoperative pain control in patients undergoing unilateral inguinal hernia repair, a comparative study between two approaches. BMC Anesthesiol 2019; 19: 184.

17. Öksüz G, Bilal B, Gürkan Y, Urfalioğlu A, Arslan M, Gişi G, et al. Quadratus lumborum block versus transversus abdominis plane block in children undergoing low abdominal surgery: a randomized controlled trial. Reg Anesth Pain Med 2017; 42: 674-9.

18. Akerman M, Pejčić N, Veličković I. A review of the quadratus lumborum block and ERAS. Front Med (Lausanne) 2018; 5: 44.

19. Carline L, McLeod GA, Lamb C. A cadaver study comparing 
spread of dye and nerve involvement after three different quadratus lumborum blocks. Br J Anaesth 2016; 117: 387-94.

20. Yang HM, Park SJ, Yoon KB, Park K, Kim SH. Cadaveric evaluation of different approaches for quadratus lumborum blocks. Pain Res Manag 2018; 2018: 2368930. 